\title{
UNIVERSITY OF GRANADA RADIOCARBON DATES IV
}

CECILIO GONZALEZ-GOMEZ, PURIFICACION

SANCHEZ-SANCHEZ, and ELENA VILLAFRANCA-SANCHEZ

Laboratorio de Datación por Carbono-14 and Instituto Andaluz de

Geología Mediterránea del CSIC Facultad de Ciencias, Universidad de Granada, 18071 Granada, Spain

This date list contains the results of ${ }^{14} \mathrm{C}$ determinations of archaeologic samples from Spain and Portugal obtained at the Laboratory mostly from 1983 to July 1986. Preparation and measurements were made in the same manner as previously reported ( $\mathrm{R}, 1982$, v 24, no. 2, p 217-221; R, 1985, v 27, no. 3, p 610-615; R, v 28, no. 3, p 1200-1205).

The published intervals of corrected data for samples younger than 7250 BP are derived from Klein et al (1982) and appear as "cal" ages. Sample descriptions are based on information provided by submitters. Age determinations and research were done with the help of Research Project 0925/81, CAICYT, Spain.

\section{ARCHAEOLOGIC SAMPLES}

Spain

\section{Castillo de Escobedo series}

Charcoal from Castillo de Escobedo ( $43^{\circ} 24^{\prime} 12^{\prime \prime} \mathrm{N}, 4^{\circ} 10^{\prime} 02^{\prime \prime} \mathrm{W}$ ), Camargo, Cantabria prov. Samples coll and subm 1983 by R BohigasRoldán, Mus Reg Prehist y Arqueol Santander, to date a humic level of dense ocupation.

UGRA-180. No. 2

$930 \pm 110$

Cal AD 890-1265.

UGRA-182. H-O N1 SE

$1190 \pm 110$

$20 \mathrm{~cm}$ depth; cal AD 610-1030.

General Comment: dates agree with expected ages.

UGRA-184. No. 1

$860 \pm 130$

Wood from Torrejón-Las Henestrosas ( $42^{\circ} 52^{\prime} 30^{\prime \prime} \mathrm{N}, 4^{\circ} 12^{\prime} 05^{\prime \prime} \mathrm{W}$ ), Las Henestrosas, Cantabria prov. Sample coll 1984 by A SobremazasSalcines and subm 1984 by R Bohigas-Roldán, to date occupation level of deposit. Comment: date agrees with expected age; cal AD 905-1325.

\section{Cova Santa series}

Charcoal from Cova Santa ( $\left.38^{\circ} 48^{\prime} 20^{\prime \prime} \mathrm{N}, 0^{\circ} 53^{\prime} 30^{\prime \prime} \mathrm{W}\right)$, Fuente La Higuera, Valencia prov. Samples coll 1978 and subm 1983 by J AparicioPérez, Dept Hist Antigua, Fac Geog \& Hist, Univ Valencia, to date Eneolithic and Neolithic periods.

UGRA-188. LL3.C-5

$5060 \pm 110$

Cal 4110-3650 BC. 
UGRA-192. LL4.C-2

$2640 \pm 120$

$90 \mathrm{~cm}$ depth; cal 1105-520 BC.

UGRA-193. LL4.C-3

$3260 \pm 900$

90cm depth; cal 2890-400 BC.

UGRA-196. LL4.C-6

$14,600 \pm 300$

$20 \mathrm{~cm}$ depth.

General Comment: age for LL4.C-6 is probably too old.

\section{Cueva del Toro series}

Charcoal from Cueva del Toro $\left(37^{\circ} 01^{\prime} \mathrm{N}, 4^{\circ} 25^{\prime} \mathrm{W}\right)$, Sierra del Torcal, Málaga prov. Samples coll and subm 1983 by D Martín-Socas, Dept Arqueol \& Prehist, Fac Geog \& Hist, Univ La Laguna, Tenerife, Canary Islands, to date Neolithic and Eneolithic periods in Málaga prov.

UGRA-189. No. 1

$3090 \pm 130$

93cm depth; cal 1670-990 BC.

UGRA-194. No. 6

$6400 \pm 280$

$2.51 \mathrm{~m}$ depth; cal 5775-4800 BC.

General Comment: dates agree with expected ages.

\section{Piedrafita series}

Charcoal from Piedrafita $\left(43^{\circ} 26^{\prime} 32^{\prime \prime}\right.$ N, $\left.5^{\circ} 59^{\prime} 07^{\prime \prime} \mathrm{W}\right)$, Las Regueras, Oviedo prov, Asturias. Samples coll 1980 by M A Blas-Cortina and subm 1983 by J Fortea-Pérez, Dept Prehist, Fac Geog \& Hist, Univ Oviedo, to date Megalithic chronology in Asturias.

UGRA-191. No. 3-PFV.1

$2610 \pm 110$

$35 \mathrm{~cm}$ depth; cal 1035-430 BC.

UGRA-195. No. 4-PFI.2

$2560 \pm 110$

$80 \mathrm{~cm}$ depth; cal 905-410 BC.

General Comment: expected age was ca 4500 BP for both samples.

UGRA-197. M-1. Villar 1

$2590 \pm 100$

Charcoal from Cerro de los Encaños (40 $03^{\prime} 20^{\prime \prime} \mathrm{N}, 2^{\circ} 26^{\prime} 40^{\prime \prime} \mathrm{W}$ ), Villar del Horno, Cuenca prov. Sample coll 1983 by A Gómez-Ruíz and subm 1984 by M Sierra-Delage, Mus Nac Etnolog, Madrid. Sample from $80 \mathrm{~cm}$ depth. Comment: date agrees with expected age; cal 1010-425 BC.

\section{Fuente de la Mota series}

Wood from Fuente de la Mota (39 39' 18' N, 2 03' W), Barchín Hoyo, Cuenca prov. Samples coll 1981 and subm 1984 by M Sierra-Delage. 
UGRA-199. 4F (81)

$55 \mathrm{~cm}$ depth; cal 770 BC-AD 425.

UGRA-202. $3 \mathrm{H} \mathrm{(81)}$

$2360 \pm 90$

$35 \mathrm{~cm}$ depth; cal 770-205 BC.

General Comment: dates agree with expected ages.

\section{Cova Matutano series}

Charcoal from Cova Matutano $\left(40^{\circ} 06^{\prime} 50^{\prime \prime} \mathrm{N}, 0^{\circ} 02^{\prime} \mathrm{E}\right)$, Villafamés, Castellón de la Plana prov. Samples coll 1984 and subm 1985 by F GusiJener, Research Service Archaeol \& Prehist County Council, Castellón de la Plana, to date Upper Paleolithic in Castellón de la Plana prov.

UGRA-201. Mat.84.2.N-IC7

$12,460 \pm 180$

UGRA-208. 8bis-N.1C5.P.260

$13,220 \pm 270$

$2.6 \mathrm{~m}$ depth.

UGRA-225. 9-10-N.1C8

$13,370 \pm 260$

$3.2 \mathrm{~m}$ depth.

UGRA-241. 3-N.1C.P.219

$11,590 \pm 150$

$2.19 \mathrm{~m}$ depth.

UGRA-242. 6-N.1C3.P.235-244

$11,570 \pm 210$

2.35 to $2.44 \mathrm{~m}$ depth.

UGRA-243. 4-N.1C1.P.220

$11,410 \pm 610$

$2.2 \mathrm{~m}$ depth.

UGRA-244. 7-N.1C4.P.235-244

$12,520 \pm 350$

2.35 to $2.44 \mathrm{~m}$ depth.

General Comment: dates agree with expected ages.

\section{Sierra Plana de la Borbolla series}

Charcoal from Sierra Plana de la Borbolla $\left(43^{\circ} 23^{\prime} 24^{\prime \prime} \mathrm{N}, 4^{\circ} 37^{\prime} 41^{\prime \prime}\right.$ W), Buelna, Llanes, Oviedo prov, Asturias. Samples coll and subm 1983 by P Arias-Cabal, Dept Prehist, Univ Santander, to date Chalcolithic and Megalithic periods in $\mathrm{N}$ Iberian peninsula.

UGRA-205. SV 83D 0-7 N 2

$580 \pm 80$

$15 \mathrm{~cm}$ depth; cal AD 1265-1425.

UGRA-206. SV 83 D N 6-7 N 2

$600 \pm 90$

$15 \mathrm{~cm}$ depth; cal AD 1250-1420. 
UGRA-209. SV 82 C E2/3 N 1C

$7550 \pm 190$

General Comment: expected ages were ca 4000 вP.

\section{Hoya Quemada series}

Charcoal from Hoya Quemada $\left(40^{\circ} 14^{\prime} 55^{\prime \prime} \mathrm{N}, 0^{\circ} 44^{\prime} 01^{\prime \prime} \mathrm{W}\right)$, Mora de Rubielos, Teruel prov. Samples coll and subm 1983 by F Burillo-Mozota, Colegio Univ Teruel, to date Bronze Age in Aragon.

UGRA-207. No. 1 C.5I-13

$4070 \pm 190$

$2.19 \mathrm{~m}$ depth; cal 2990-2325 BC.

UGRA-211. No. 2 C.5I-5

$3260 \pm 100$

2.23m depth; cal 1770-1370 BC.

UGRA-212. No. 2 C.11 I

$3450 \pm 90$

$0.70 \mathrm{~m}$ depth; cal 2055-1595 BC.

UGRA-213. No. 4 C.11 H

$3420 \pm 100$

$1.61 \mathrm{~m}$ depth; cal 1980-1555 BC.

General Comment: expected age for UGRA-207 was 3400 BP.

UGRA-214. MBI/II/1

$2210 \pm 110$

Charcoal from El Jardinero $\left(\begin{array}{llllll}39^{\circ} & 23^{\prime} & \mathrm{N}, & 7^{\circ} & 10^{\prime} \mathrm{W}\end{array}\right)$, Valencia de Alcántara, Cáceres prov. Sample coll and subm 1983 by P Bueno-Ramírez, Dept Prehist, Univ Alcalá de Henares, Madrid, from $2.67 \mathrm{~m}$ depth. Comment: date agrees with expected age; cal 570-5 BC.

\section{Cerro de la Mora series}

Charcoal from Cerro de la Mora ( $\left.37^{\circ} 13^{\prime} 31^{\prime \prime} \mathrm{N}, 3^{\circ} 59^{\prime} 07^{\prime \prime} \mathrm{W}\right)$, Moraleda, Granada prov. Samples coll and subm 1985-1986 by J CarrascoRús and M Pastor-Muñoz, Dept Prehist, Univ Granada, to date end of Bronze Age in Granada prov.

UGRA-217. TABLA 12

$3690 \pm 90$

$2.20 \mathrm{~m}$ depth; cal 2470-1870 BC.

UGRA-218. CAPA 10

$2920 \pm 90$

2m depth; cal 1385-855 BC.

UGRA-231. TABLA 3

$2670 \pm 100$

$1 \mathrm{~m}$ depth; cal 1085-610 BC.

UGRA-232. CAPA 9

$2670 \pm 90$

$1.60 \mathrm{~m}$ depth; cal 1070-615 BC.

$2740 \pm 90$

UGRA-235. CAPA 42

$7 \mathrm{~m}$ depth; cal 1200-675 BC. 
UGRA-262. Fortificación

From 2.40 to 3m depth; cal 2475-1885 BC.

UGRA-263. CM/86/CV-B

$2990 \pm 90$

3m depth; cal 1495-905 BC.

UGRA-264. CM/V-869 (Botón)

$2850 \pm 90$

$2.90 \mathrm{~m}$ depth; cal 1320-810 BC.

General Comment: dates agree with expected ages.

\section{Los Castillejos de Sanchorreja series}

Charcoal from Los Castillejos de Sanchorreja $\left(40^{\circ} 39^{\prime} 40^{\prime \prime} \mathrm{N}, 4^{\circ} 52^{\prime} 20^{\prime \prime}\right.$ W), Sanchorreja, Avila prov. Samples coll 1981 and subm 1984 by F J González-Tablas-Sastre, Dept Prehist \& Arqueol, Univ Salamanca, to date end of Bronze Age in N Meseta.

\section{UGRA-237. C-1.VI}

$3670 \pm 100$

$1.60 \mathrm{~m}$ depth; cal 2390-1774 BC.

UGRA-238. C-1.V

$\mathbf{3 7 7 0} \pm 90$

$1.20 \mathrm{~m}$ depth; cal 2530-1945 BC.

General Comment: ages probably a little older than expected.

UGRA-254. SCH-80/D-12

$6160 \pm 100$

Charcoal from Cueva Chica de Santiago $\left(38^{\circ} 00^{\prime} 48^{\prime \prime}\right.$ N, $\left.5^{\circ} 55^{\prime} 07^{\prime \prime} \mathrm{W}\right)$, Cazalla de la Sierra, Sevilla prov. Sample coll 1980 and subm 1983 by P Acosta-Martínez, Dept Prehist \& Arqueol, Univ Sevilla, to date start of Neolithic period in Sierra Morena. Sample from $2.70 \mathrm{~m}$ depth. Comment: expected age was ca 7000 BP; cal 5325-4910 BC.

\section{Cueva de Nerja series}

Seeds and charcoal from Cueva de Nerja $\left(36^{\circ} 45^{\prime} 45^{\prime \prime} \mathrm{N}, 3^{\circ} 50^{\prime} 55^{\prime \prime} \mathrm{W}\right)$, Maro, Nerja, Málaga prov. Samples coll 1980-1982 and subm 1983 by M Pellicer-Catalán, Dept Prehist \& Arqueol, Univ Sevilla, to date Chalcolithic and start of Neolithic periods in Andalusian coast.

UGRA-255. NM-80/B/2

$5190 \pm 90$

$0.70 \mathrm{~m}$ depth; cal 4295-3780 BC.

UGRA-261. NT-82/9

$6200 \pm 100$

$1.40 \mathrm{~m}$ depth; cal 5360-4925 BC.

General Comment: expected age for UGRA-261 was ca 7000 BP.

UGRA-259. DE-81/13-NW

$6260 \pm 100$

Charcoal from Cueva de la Dehesilla (36 $\left.39^{\prime} 45^{\prime \prime} \mathrm{N}, 5^{\circ} 38^{\prime} 40^{\prime \prime} \mathrm{W}\right)$, Algar, Cádiz prov. Sample coll 1981 and subm 1983 by P Acosta-Martínez, to date start of Neolithic period in S Iberian peninsula. Sample from $3.60 \mathrm{~m}$ depth. Comment: expected age was ca 7500 BP; cal 5455-4960 BC. 
UGRA-260. C.T.I/1

$3530 \pm 70$

Bones from Sepulcro 1 Cortijo del Tardón $\left(37^{\circ} 01^{\prime} \mathrm{N}, 4^{\circ} 33^{\prime} \mathrm{W}\right)$, Antequera, Málaga prov. Sample coll and subm 1986 by J E Ferrer-Palma, Dept Prehist \& Arqueol, Fac Filosof \& Letras, Univ Málaga, to date Megalithic culture in Málaga prov. Comment: expected age was ca 4000 BP; cal 2140 $1690 \mathrm{BC}$.

UGRA-233. 6-C.10E-S.6

$3760 \pm 100$

Charcoal from Cueva del Coscojar $\left(40^{\circ} 17^{\prime} 00^{\prime \prime} \mathrm{N}, 0^{\circ} 41^{\prime} 10^{\prime \prime} \mathrm{W}\right)$, Mora de Rubielos, Teruel prov. Sample coll 1982 and subm 1983 by F BurilloMozota, Colegio Univ Teruel, to date Middle Bronze Age and Eneolithic period in Levante and Ebro valley. Comment: expected age was ca $2600 \mathrm{BP}$; cal 2530-1910 BC.

\section{Portugal}

\section{Castelo de Aguiar series}

Charcoal from Castelo de Aguiar ( $\left.41^{\circ} 28^{\prime} 07^{\prime \prime} \mathrm{N}, 7^{\circ} 38^{\prime} 17^{\prime \prime} \mathrm{W}\right)$, Teloes, Vila Pouça de Aguiar, Tras os Montes prov. Samples coll and subm 1983 by S M Soares-Oliveira, Inst Arqueol, Fac Letras, Univ Porto, to date recent prehist in $\mathrm{N}$ Portugal.

UGRA-179. CST AGR 83-2

$4700 \pm 100$

2m depth; cal 3775-3170 BC.

UGRA-181. CST AGR 83-4

$2730 \pm 140$

$1.10 \mathrm{~m}$ depth; cal 1245-610 BC.

UGRA-185. CST AGR 83-3

$3930 \pm 180$

$1.10 \mathrm{~m}$ depth; cal 2900-2015 BC.

General Comment: age for CST AGR 83-4 is probably a little younger.

UGRA-203. No. 7

$2550 \pm 100$

Seeds from Castro de Romariz ( $\left.41^{\circ} 51^{\prime} 52^{\prime \prime} \mathrm{N}, 8^{\circ} 37^{\prime} 37^{\prime \prime} \mathrm{W}\right)$, Romariz, Vila da Feira, Aveiro prov. Sample coll 1982 by R M Sobral-Centeno and subm 1984 by A Coelho-Ferreira-da-Silva, Fac Letras, Univ Porto. Comment: date agrees with expected age; cal 865-415 BC.

UGRA-221. 1/CU-84/5.V/G2

$2340 \pm 100$

Charcoal from Corno do Cunho ( $\left.41^{\circ} 17^{\prime} 02^{\prime \prime} \mathrm{N}, 6^{\circ} 31^{\prime} 55^{\prime \prime} \mathrm{W}\right)$, Peredo da Bemposta, Modagorico, Tras os Montes prov. Sample coll by P J SoaresFelix and subm 1984 by M De-Jesus-Sanches, Grup Est Arqueol do Porto, from $60 \mathrm{~cm}$ depth. Comment: expected age was ca $3200 \mathrm{BP}$; cal 770-180 BC.

UGRA-222. No. 9

$1440 \pm 100$

Charcoal from Citania de Sanfins ( $41^{\circ} 19^{\prime} 22^{\prime \prime}$ N, $8^{\circ} 22^{\prime} 52^{\prime \prime}$ W), Sanfins 
de Ferreira, Paços de Ferreira, Porto prov. Sample coll 1979 and subm 1984 by A Coelho-Ferreira-da-Silva, from $60 \mathrm{~cm}$ depth. Comment: expected age was 1850 BP; cal AD 415-770.

\section{Monte da Tumba series}

Charcoal from Monte da Tumba (38 $\left.16^{\prime} 54^{\prime \prime} \mathrm{N}, 8^{\circ} 13^{\prime} 12^{\prime \prime} \mathrm{W}\right)$, Torrão, Alcacer do Sal, Baixo Alemtejo prov. Samples coll 1982 and subm 1983 by M J Coelho-Soares, Univ Evora and Mus Arqueol Setúbal, to date Chalcolithic period in SW Iberian peninsula.

UGRA-234. M.T./Cv.1

$4280 \pm 100$

Cal 3350-2650 BC.

UGRA-236. M.T./Cv.3

$4550 \pm 150$

Cal 3655-2915 вс.

General Comment: dates agree with expected ages.

GEOLOGIC SAMPLES

Spain

UGRA-210. ZL-860 B

$>\mathbf{2 0 , 0 0 0}$

Charcoal from Lanjarón ( $36^{\circ} 55^{\prime} 20^{\prime \prime} \mathrm{N}, 3^{\circ} 28^{\prime} 50^{\prime \prime} \mathrm{W}$ ), Granada prov. Sample coll and subm 1983 by L García-Rossell, Inst Andaluz Geol Mediterranean CSIC, Fac Cien, Univ Granada, to date Quaternary fm. Sample from $7 \mathrm{~m}$ depth.

UGRA-253. Moucide-2

$1530 \pm 80$

Charcoal from Moucide ( $\left.43^{\circ} 35^{\prime} 30^{\prime \prime} \mathrm{N}, 7^{\circ} 23^{\prime} 25^{\prime \prime} \mathrm{W}\right)$, Burela, Lugo prov. Sample coll and subm 1985 by F Díaz-Fierros-Viqueira, Dept Edafol, Fac Farm, Univ Santiago de Compostela. Sample from 1.05m depth. Comment: date much younger than expected; cal AD 300-620.

\section{Saldropo series}

Peat from Saldropo $\left(43^{\circ} 03^{\prime} 10^{\prime \prime} \mathrm{N}, 2^{\circ} 43^{\prime} 36^{\prime \prime} \mathrm{W}\right)$, Ceanuri, Vizcaya prov. Samples coll and subm 1985 by F M Ugarte-Elorza, Soc Cien Aranzadi, Mus S Telmo, San Sebastián, to date Vasco-Cantabric country.

UGRA-256. No. 1

$4510 \pm 110$

$2.70 \mathrm{~m}$ depth; cal 3545-2925 BC.

UGRA-257. No. 3

$920 \pm 100$

$0.30 \mathrm{~m}$ depth; cal AD 900-1260.

UGRA-258. No. 2

$2460 \pm 100$

$1.30 \mathrm{~m}$ depth; cal 805-390 BC.

General Comment: dates agree with expected ages. 
REFERENCES

González-Gómez, C, López-González, J D and Domingo-García, M, 1982, University of Granada radiocarbon dates I: Radiocarbon, v 24, no. 2, p 217-221.

González-Gómez, C, Sánchez-Sánchez, P and Domingo-García, M, 1985, University of Granada radiocarbon dates II: Radiocarbon, v 27, no. 3, p 610-615.

González-Gómez, C, Sánchez-Sánchez, P and Villafranca-Sánchez, E, 1986, University of Granada radiocarbon dates III: Radiocarbon, v 28, no. 3, p 1200-1205.

Klein, S, Lerman, J C, Damon, P E and Ralph, E K, 1982, Calibration of radiocarbon dates: Tables based on the consensus data of the Workshop on Calibrating the Radiocarbon Time Scale: Radiocarbon, v 24, no. 2, p 103-150. 